\title{
NEUROGLIAL RELATIONSHIPS IN THE HUMAN RETINAL CAPILLARY NETWORK*
}

\author{
BY
}

\author{
C. A. ERSKINE
}

School of Anatomy, Trinity College, Dublin

THE investigation described in this paper was designed to throw light on one aspect of the vascular relationships in the human posterior retina; such investigations contribute to the more ambitious attempts to solve the difficult and obscure problem presented by the vascular changes in diabetic retinopathy. New and fruitful ideas on retinal structure, function, and pathology have been put forward in the studies of this serious disorder by Ashton (1959), Bloodworth (1962), and Cogan, Toussaint, and Kuwabara (1961). In particular, Cogan has emphasized the important functional relationships of Müller's cells; in the retina these modified astrocytes provide a clear example of the supporting function of neuroglia; their interrelationship with the capillary circulation in glucose metabolism has been shown to be crucial for retinal function.

Special interest therefore attaches to detailed studies on the intimate structural relationships of the capillary endothelium in the posterior retina and to regional differences in glial distribution in relation to the capillary networks. In retinal research, the numerous cell types, complex interrelationships, and differences in characteristics from many central nervous cells, make new methods or modifications of standard techniques for nerve tissue of special importance in the determination of glial cell type and relationships and indicate the need for caution in interpretation.

\section{Material and Methods}

Examination of sample sections was undertaken in a selection of eleven enucleated human eyes to find normal retinae and to determine satisfactory fixation; three of these specimens were suitable for detailed examination for their neurogliavascular relationships. The study was confined to typical capillary areas of the posterior retina by the use of two main methods and their modifications; systematic examination of the whole retina was not attempted. Control sections were provided by reference series of whole normal human retinae at various ages.

Fixation was carried out for 2 days and for 10 days in isotonic formol saline (as recommended for central neurons by Cammermeyer) and in formalin ammonium bromide for Cajal's gold sublimate method for astrocytes.

\section{Standard Methods}

(1) Serial sections at $2 \mu$ were made from paraffin blocks of 4-5 mm. ${ }^{2}$ pieces of posterior polar retina out to $20^{\circ}$ from the nerve head; sections were mounted in aqueous glycerine for phase contrast photomicrography.

* Read at the combined meeting of the British Diabetic Association and the Irish Ophthalmological Society in Dublin, April 26, 1963. 
(2) Pieces of 4-5 mm. ${ }^{2}$ of the same retina were serial sectioned at $8 \mu$ and impregnated by Cajal's gold sublimate method for astrocytes.

\section{Modifications}

(1) Several 2-day isotonic formalin-fixed pieces were washed in distilled water and in 1 per cent. ammonia, incubated in 0.3 per cent. sodium hydroxidepancreatin (Langeron, 1942) for 6 hours at $37^{\circ} \mathrm{C}$, teased with glass needles, and mounted as temporary preparations in glycerine medium for phase-contrast photomicrography of capillary sections and perivascular structure.

(2a) Isotonic formalin-fixed pieces of retina of $4-5 \mathrm{~mm} .^{2}$ were incubated as above and kept for 2 hours in 10 per cent. hydrobromic acid at $37^{\circ} \mathrm{C}$; when washed, each whole piece was impregnated by Cajal's gold sublimate method as for frozen sections, blocked, and serially sectioned at $8 \mu$.

(2b) Comparative adjacent sections were made from the formalin ammonium bromide pieces impregnated by del Rio Hortego silver carbonate method.

(2c) Sections of $8 \mu$ stained by Mallory's phosphotungstic acid haematoxylin (adapted for formalin fixation) and nearly decolourized were used for oil immersion optical section in the identification of oligodendrocyte nuclei.

(3) Small pieces (1-2 mm. $\left.{ }^{2}\right)$ of unfixed retina were microdissected in Earle's balanced salt solution to study by phase-contrast microscopy the number and attachment of astrocyte end-feet on the capillary endothelium.

\section{Observations}

\section{(1) Superficial Capillary Network}

(a) Müller's Astroglia (radial fibres).-When impregnated by the modified Cajal's method (sodium hydroxide-pancreatin) the thick irregular cytoplasmic sheath was seen to cover completely the capillary in the nerve fibre layer (Fig. 1). Numerous fine granules appeared in the processes and

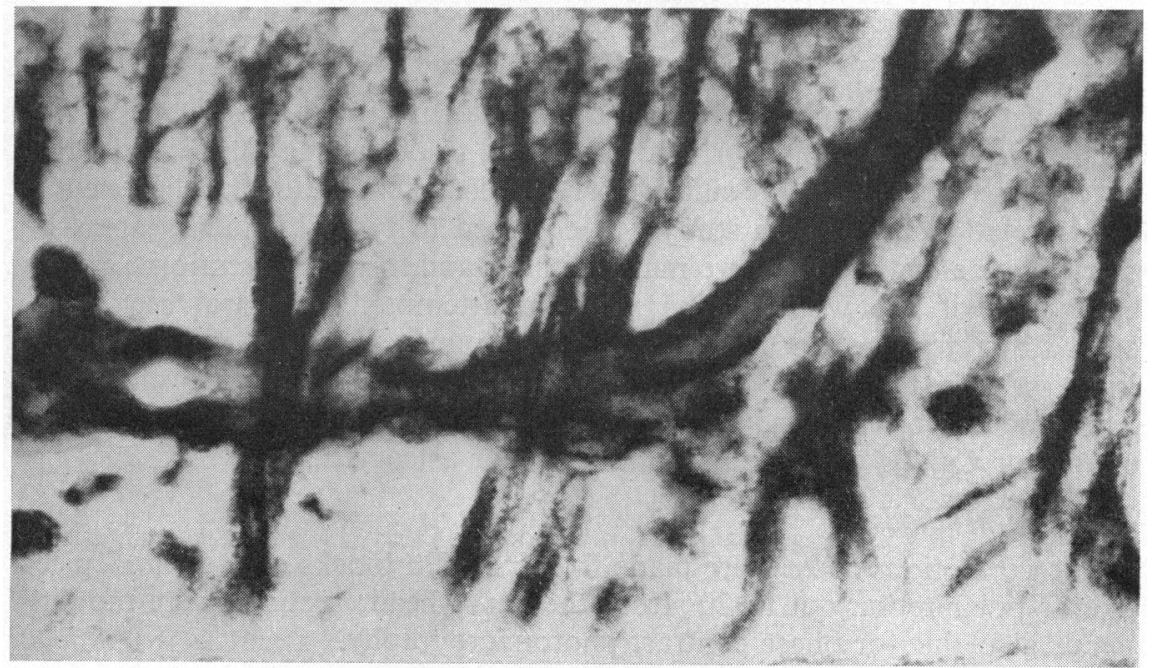

FIG. 1.-Superficial capillary surrounded by Müller's cytoplasm; many protoplasmic processes contain fine granules. Modified gold sublimate method. $\times 1,350$. 
in the loose cytoplasmic veil surrounding the endothelium; investment was continued into the beginning of the linking vessels as they pass down to the immediately adjacent ganglionic layer. Definite perivascular astroglia were not visible on these vessels in the areas impregnated. (Variability in impregnation within each treated piece occurred by this method which was therefore unsuitable for systematic study.)

(b) Cells of the Ganglionic Layer.-By phase-contrast microscopy thin sections at this level showed well-preserved typical ganglion cells with granular cytoplasm (Fig. 2c). In addition, smaller elements distinct from the so-called small ganglion cells were seen; these had oval dark nuclei containing several dark granules, a dark cytoplasm, and a bright phase-contrast halo. Their perivascular relationships (Fig. $2 a, b$ ) were similar to those of oligodendrocytes on central capillaries.

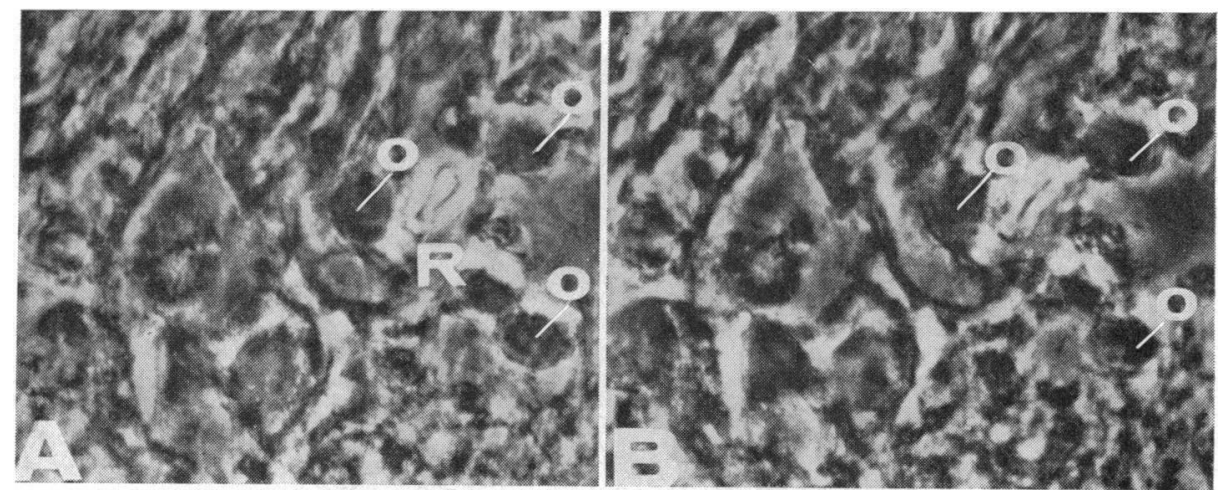

FIG. 2(a,b).-Two-stage serial section of outer part of a capillary loop in ganglionic layer with small dark cells in perivascular position.

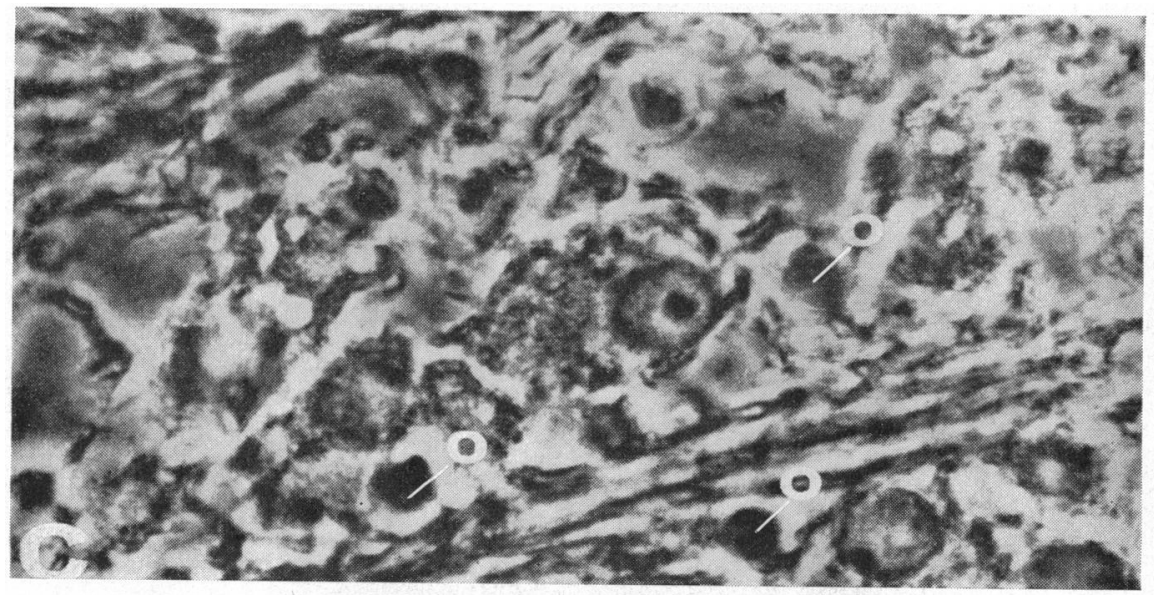

FIG. 2(c).-Ganglionic layer in thin section $(2 \mu)$ with well-preserved nuclei and cytoplasm; small dark glial cells (0) present. (R: red blood cell). Phase contrast. $\times 1,350$. 
In decolourized Mallory phase-contrast microscopy (Fig. 3), their nuclei were characteristic of central oligodendrocytes; they also appeared grouped in satellite positions in relation to neurons as in the central nervous

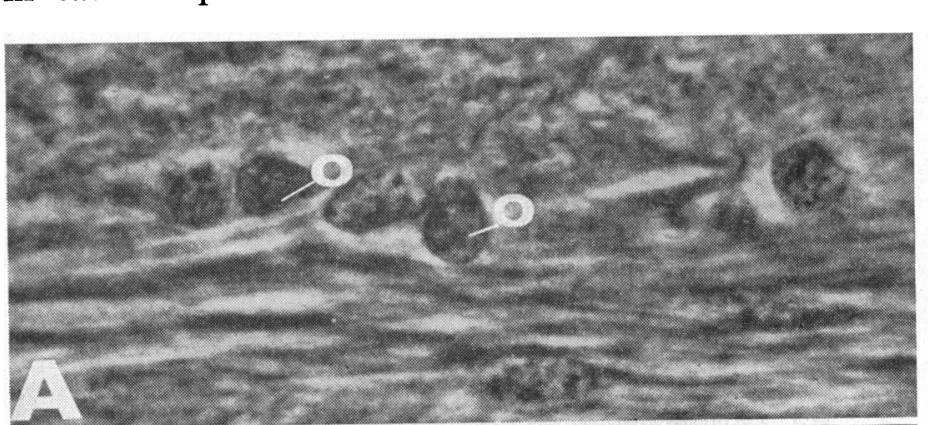
system. Except for the small extent of the thick ganglion layer near the macula, where they are more difficult to distinguish, these relationships obtained over the

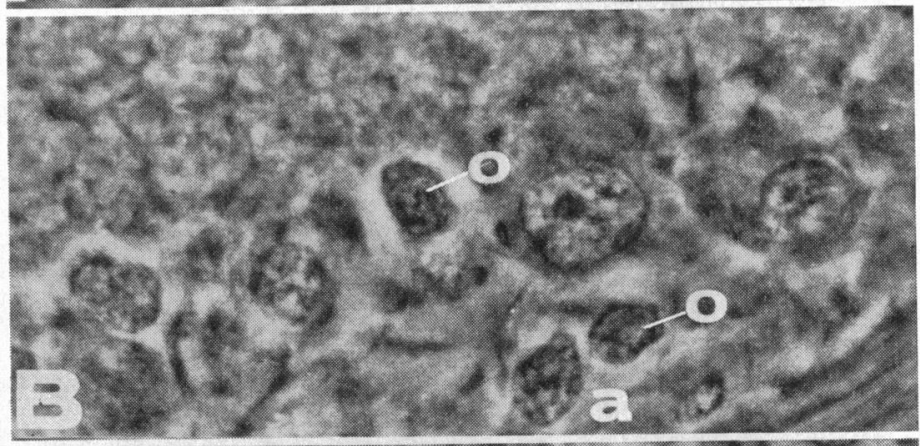
greater part of the posterior retina.

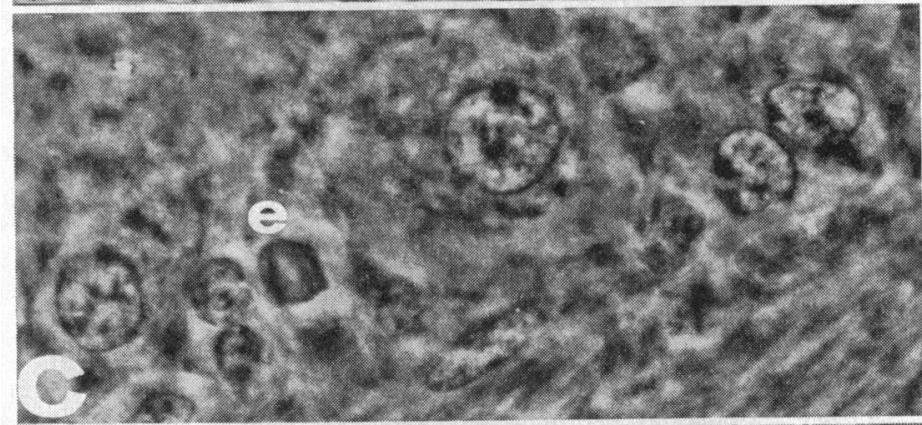

Fig. $\quad 3(a)$. - Outer margin capillary in ganglion cell layer. Perivascular oligodendrocyte and astrocyte

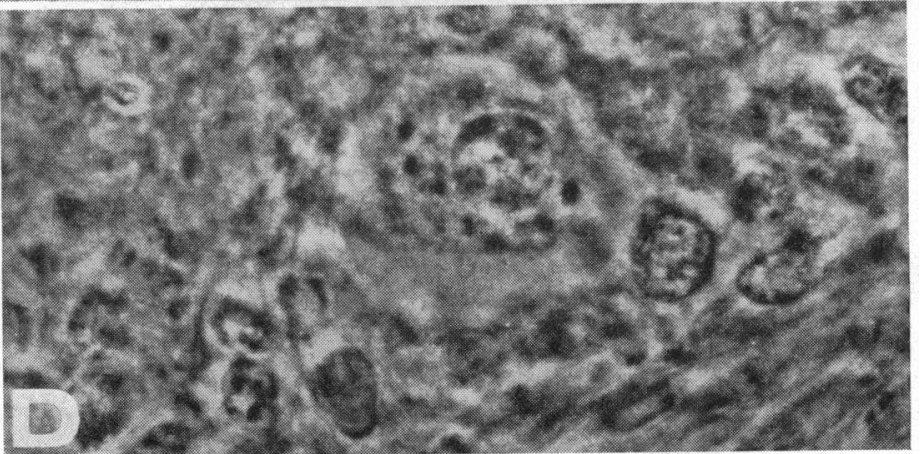
nuclei.

Fig. 3(b,c,d).-Optical section through ganglion cell layer and segment of capillary; astrocyte and perivascular and satellite oligodendrocytes close to large central ganglion cell (A: astrocyte; $\mathrm{O}$ : oligodendrocyte; E: astrocyte end-foot). Decolourized Mallory stain phase-contrast method. $\times 1,500$. 
(c) Astrocytes of the Ganglionic Layer.-By Cajal's method a few single astrocytes may be seen in close relation to the capillary wall (Fig. $4 a, b$ ).

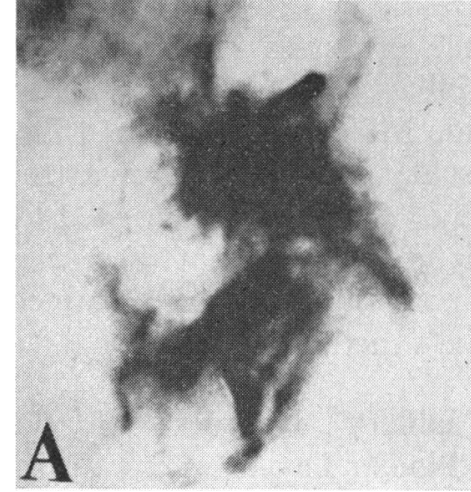

FIG. 4(a).-Astrocyte by gold impregnation. $8 \mu$ section. $\times 2,500$.

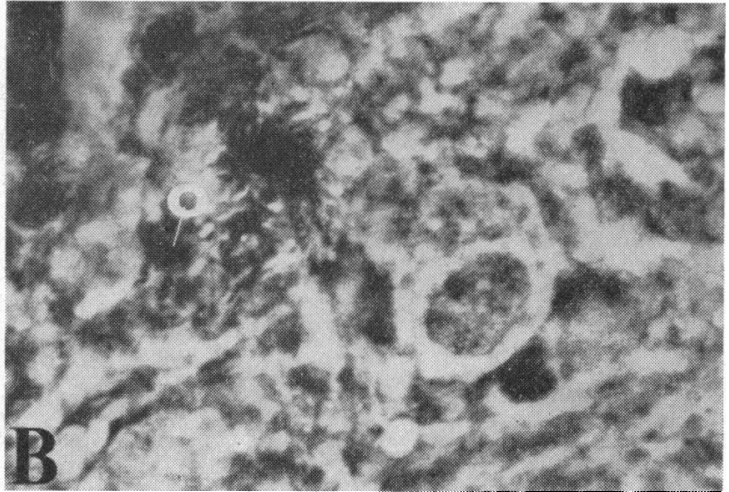

FIG. 4(b).-Same area (in wider field) as (a) by phasecontrast, showing additional details and small glial cell close to the astrocyte. (Ganglion cell on right). $\times 1,125$.

Problems of identification of individual components were shown by comparing these with similar elements classified as horizontal cells by Polyak (1957). Comparative phase-contrast examination of the same preparation revealed smaller cells which were unstained by Cajal's method. (In Fig. $4 b$ one is seen in close relation to the astrocyte; it is much smaller than the ganglion cell to which the astrocyte can be seen to send processes.)

\section{(2) Linking Capillaries}

Phase-contrast examination of longitudinal sections through these capillaries from the nerve fibre layer through the inner plexiform layer (Fig. 5)

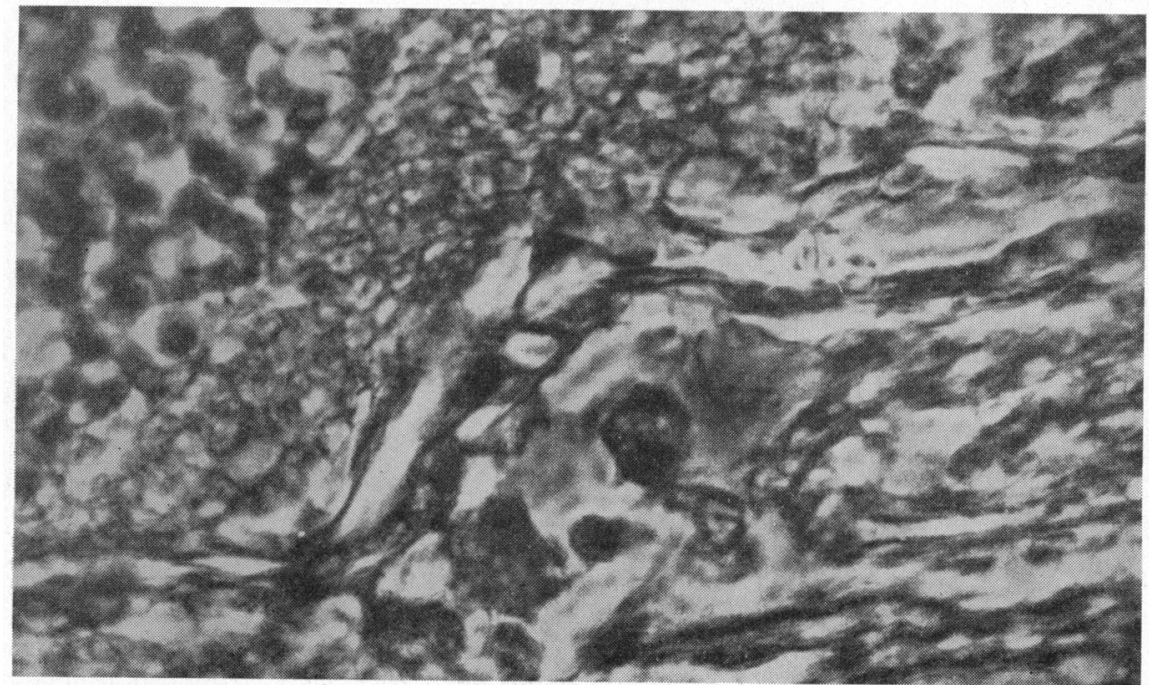

FIG. 5.- Linking capillary by phase-contrast, showing small dark elements with thick processes closely applied to capillary. $\times 600$. 
showed a number of dark glial processes in relation to elements which

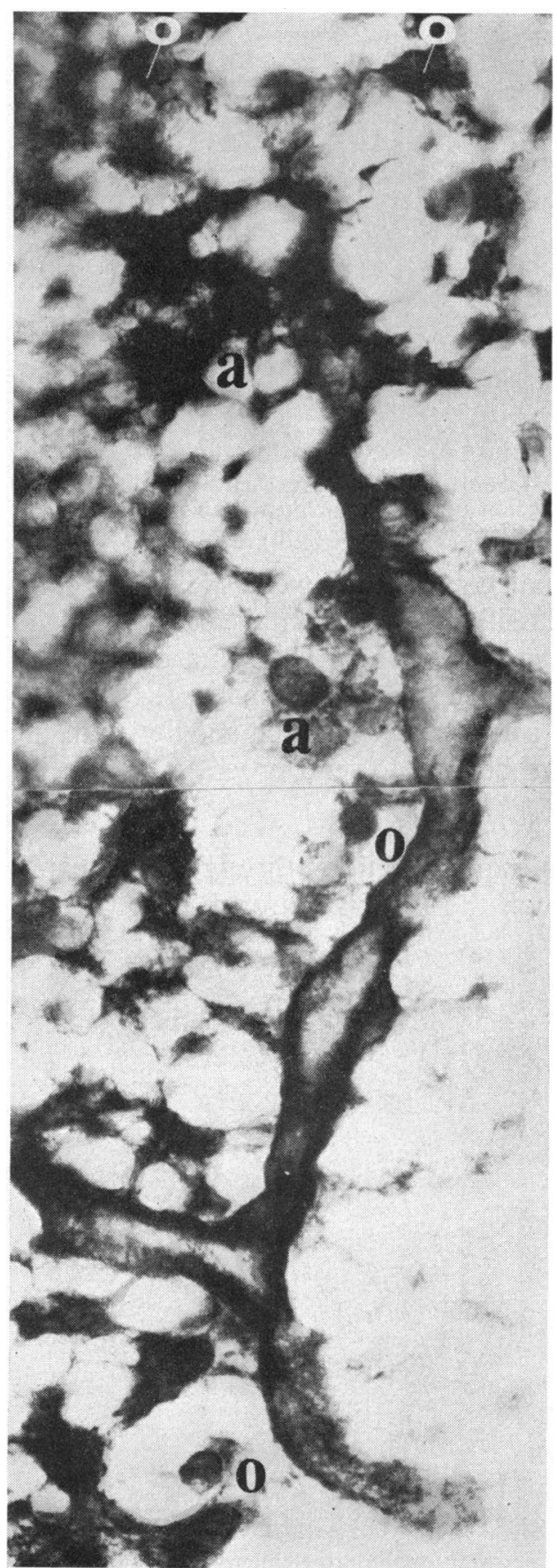
suggested perivascular cells closely applied to the capillary endothelium; the processes ran parallel to the capillary walls; they appear to be inside Müller's cytoplasm. The perivascular cells classified as astrocytes by Wolter were suggested by this picture, except that these elements were not selectively stained by Cajal's method.

\section{(3) Capillary Network of the Bipolar Layer}

(a) Gold Sublimate Method (modified).-At the top of Fig. 6 two cells interpreted as oligodendrocytes are present. In a few parts of the capillary network a pair of astrocytes were seen. In Fig. 6 a group of three cells is visible which send thick processes to the capillary, which appears attenuated mainly because it is cut through its outer margin, though shrinkage is also present. These capillaries showed a glial sheath which was much less thick than that found in the nerve fibre layer. It formed a delicate

Fig. 6.-Outer capillary network: two oligodendrocytes at top of figure. Group of cells resembling astrocytes on left of capillary (cut through margin). Astrocyte partly impregnated in upper centre with oligodendrocyte below. Oligodendrocyte at lower end and possibly at bifurcation. Strands of cytoplasm partly due to shrinkage of capillary. Granular glial cytoplasm seen at lower end surrounding capillary. Gold impregnation. $\times 1,260$. 
membrane containing fine granules and is similar to both Müller's fibre-layer expansions and astrocytoplasm. Incompletely impregnated cells show oval nuclei with several nucleoli similar to the typical central astrocyte and an irregular granular cytoplasm which often contained a number of dispersed refractile granules. A smaller dark perivascular cell was seen near this astrocyte and the endothelium. At the bifurcation of this capillary and attached by a few processes to the endothelium were several dark stellate cells; their appearance was duplicated by phase-contrast microscopy. Some may be either glial cells or neurons of the bipolar layer; in the latter case the processes may then be interpreted as Müller's cytoplasm (accentuated by shrinkage) connecting the capillary to the cell body.

(b) Phase-contrast Microscopy and Microdissection.-End plates of astrocytes were easily seen on the capillaries of the deep network; many were clearly situated within the sheaths of Müller's cytoplasm, since they were in close contact with the capillary endothelium. They were arranged both singly and in groups. In Fig. 7 a section of the capillary is shown without end-feet; this appearance is due partly to tension on the capillary. Cytoplasm of Müller's astroglia has been pulled aside. It can be shown that end-feet were not fixed or fused to the endothelium, since they could be separated by drawing the astrocyte away from the capillary.

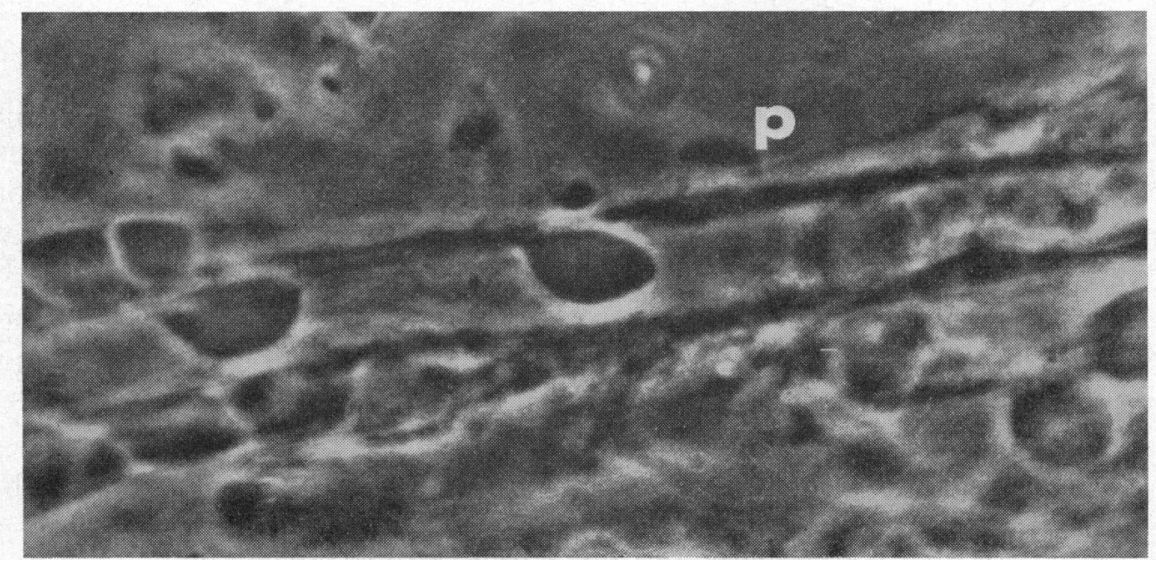

FIG. 7.-Deep capillary network: two astrocyte end-feet in plane of focus on capillary endothelium. ( $\mathrm{P}$ : pericyte nucleus). Phase-contrast. $\times 1,500$.

The end-foot remained attached to the astrocyte process as the cell was displaced (Fig. 8, overleaf).

\section{Discussion}

Individual glial elements in the central nervous system are acknowledged to be difficult to distinguish from many neurons though it is not difficult to obtain agreement on the characteristics and identity of groups; the same 


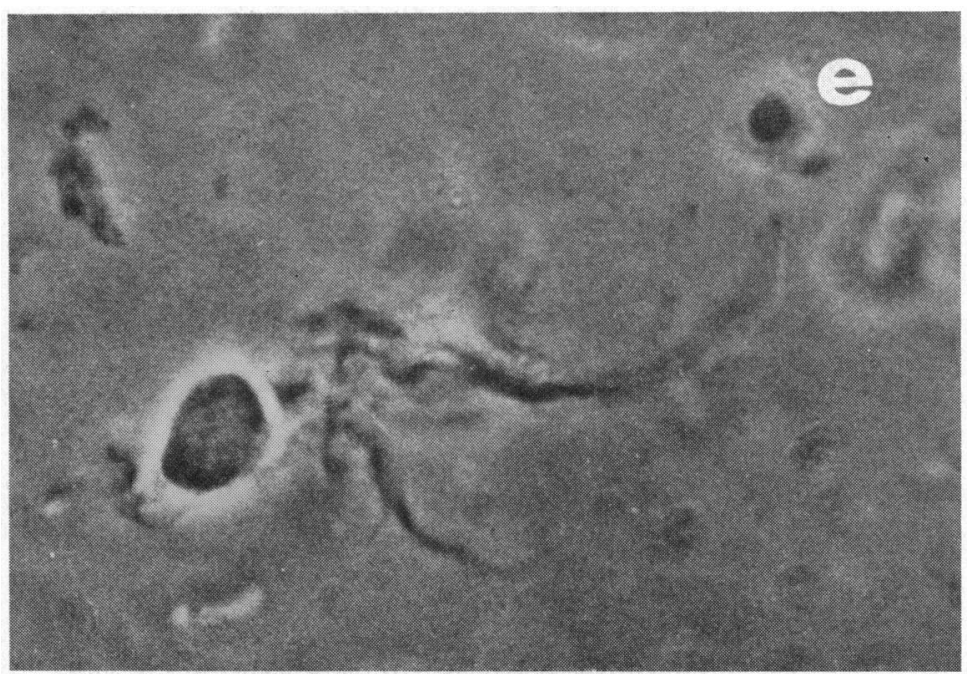

FIG. 8.-Separated astrocyte from deep network with attached end-plate from capillary of Fig. 7. Phase-contrast. $\quad \times 800$.

applies to the neuroglia of the retina. The astrocyte-capillary-neuron relations shown in the inner layer of the bipolar cells are similar to those found in the central nervous system, i.e. astrocytoplasm intervenes between the capillary and the neuron with the glia cell in close relation to both. The considerable gaps between the astrocytoplasm and end-feet at the outer bipolar layer are closed by Müller's cytoplasm. The glial membrane seems to be altogether more complete than in most parts of the central nervous system where approximately 20 per cent. of the capillary is not directly covered by glial cytoplasm.

The similarity between the cytoplasm of Müller's cells and astrocytes when incompletely impregnated by gold sublimate methods is notable and suggests similarity in function. It might be expected that the astrocytes cooperate with Müller's cells in related metabolic function (even if these functions are not identical). Thus the suggested relatively smaller number of typical astrocytes in the retina as compared to the central nervous system may be explained by astrocytic function of Müller's cells.

Polyak (1957) and Cone and MacMillan (1932) have stated that in the retina oligodendrocytes are absent as normal structures. This is to be expected, since oligodendrocytes are related to the presence of myelin, which is not present in the retina. On the other hand, Bloodworth (1962) cites Gartner (1950) to the effect that cytoid bodies in diabetic retinopathy may be degenerated oligodendrocytes, though the origin of these bodies is considered by most authors as unsettled and in a later paper Wolter (1961) regards them as of nerve fibre origin. Oligodendrocytes form such a large proportion of central cells and are so closely associated with neurons in all parts of the 
central nervous system that it would seem likely that they are represented in the retina. The appearance of cells with characteristics of central oligodendrocytes by phase-contrast microscopy may not in fact be in contradiction with the absence of myelin in the retina; in the neuropil of the central nervous system, oligodendrocytes are not directly related to myelin. Since modified astrocytes are present in the retina, modification of oligodendrocytes may not be unexpected, though their nuclear characteristics remain distinct; absence of myelin by itself may be sufficient to account for some changes in characteristics of cytoplasm and processes. A proportion of cells classified as small ganglion cells, amacrine, horizontal cells, or neurosecretory cells (Becher, 1955), though characteristic in many respects of neurons, may function as oligodendrocytes. Decolourized nuclear stain phase-contrast microscopy has shown a high proportion of typical oligodendrocyte nuclei in most of the ganglion cell layer; it also suggests that the proportion of oligodendrocyte type cells in the bipolar layer may also be large.

Normal capillary structure, pattern, and distribution have been thoroughly investigated by Michaelson (1954), Ashton (1959), Cogan and others (1961), and Bloodworth (1962). In diabetic retinopathy there are indications that degeneration takes place in all elements of the retina (Bloodworth, 1962) and that in the past too much attention has been given to the capillary. Ashton has pointed out, however, that microaneurysm formation in an otherwise normal fundus is most characteristic of diabetic retinopathy. Apart from a thick basement membrane, electron microscopy has not shown structural peculiarities in the retinal capillary. On the other hand unique relationships are found with the glia, particularly in the difference in thickness of Müller's cytoplasm in the superficial and deep network, the irregular glial cell relationships in the bipolar layers (whether the processes between the capillaries and neurons is of oligodendrocytic or Müller's cell in origin or not), and the regional distribution of astrocytes with similar relationships to the endothelium and neurons but with proportional reduction compared with the central nervous system.

No information is available at present on the effects of glial changes on the capillary endothelium. Though it would not be denied that support may be given to the capillary by retinal structures including the glia, the circumscribed development of the microaneurysm suggests focal functional reactions in the endothelial cell rather than purely mechanical changes in support. Sensitive glial elements must be among the earliest to react in retinopathy; oligodendrocytes are sensitive elements in the central nervous system and a considerable proportion of the cells of the ganglion layer of the retina appears to consist of oligodendrocytes.

Apart from the vascular relationships and distribution of individual cells which have been described, further systematic and quantitative studies on the retinal neuroglia are indicated to determine their characteristics, variations, and real numbers in different regions. 


\section{Summary}

Capillary areas from normal human retinae were examined to provide a basis for the description of the earliest changes in glial structure in diabetic retinopathy. From the posterior retinae of eleven enucleated eyes selected areas of three specimens were studied in detail for glial cell type and endothelial relationships in the different segments of the capillary network. The methods used were microdissection, modifications of metallic impregnation methods, phase-contrast microscopy of thin sections, and decolourized Mallory stained phase-contrast sections in adjacent areas. Observations included the distribution of astrocyte end-feet in direct contact with the endothelium below Müller's cytoplasm in both superficial and deep networks, marked variation in thickness in Müller's cytoplasm, and the presence of perivascular and satellite cells with typical oligodendrocyte nuclei, especially distinct in the ganglion cell layers.

AshtoN, N. (1959). Lancet, 2, 625.

\section{REFERENCES}

BECHER, H. (1955). Cited by Reiser K. A. (1959). "Handbuch der mikroskopischen Anatomie des Menchen", ed. W. von Möllendorf, band 4, teil 4, pp. 185-514. Springer, Berlin.

BloodWorth, J. M. B., JR. (1962). Diabetes, 11, 1.

Cogan, D. G., Toussaint, D., and Kuwabara, T. (1961). Arch. Ophthal., 66, 366.

CONE, W., and MACMILlan, J. A. (1932). In "Cytology and Cellular Pathology of the Nervous System", ed. W., Penfield, vol. 2, p. 389. Hoeber, New York.

GARTNer, S. (1950). Amer. J. Ophthal., 33, 727.

LANGeron, M. (1942). "Précis de microscopie", 6th ed. Masson, Paris.

Michaelson, I. C. (1954). "Retinal Circulation in Man and Animals". Thomas, Springfield, Ill.

Polyak, S. (1957). “The Vertebrate Visual System”, ed. H. Klüver. University of Chicago Press.

WOLTER, J. R. (1961). Ibid., 51, 1123. 